were examined. PCR detected 20 (1.78\%) CT cases; 20 (1.78\%) MG; $13(1.16 \%)$ TV; $3(0.27 \%)$ GC, whereas microscopy showed no TV or GC positive results. That means that screening revealed no STIs in this group at all. The study showed that the majority of women screened were aged 35-44 (44.2\%), $p<0.05$, whereby the maximum prevalence of CT was observed among 20-34-years-old women (3.5\%), p<0.001, MG among 25-34-years-old women (3.3\%), $\mathrm{p}<0.001$. We registered no significant TV or GC prevalence distribution among the age groups. It is important to note that the overall STI burden reported by this DVC for 2010 comprised of $9(0.05 \%)$ TV positive cases, no GC was detected among 16231 persons screened. The data observed in this study allows us to suggest that PCR could reveal the following amounts of STIs in this group-Ct-288 (95\% CI 159 to 418); Mg-288 (95\% CI 159 to 418); Tv-187 (95\% CI 90 to 285); Ng-43 (95\% CI 0 to 92).

Conclusions The data obtained shows the inefficacy of the routine STI screening in Russia. Low sensitivity diagnostic tools prevent us from revealing huge amounts of positive results. At the same time implementation of modern methods with higher sensitivities to the ongoing system will lead to more effective STI uncovering, especially in the groups of higher risk.

\section{P1-S1.04 THE PATTERN OF STI BURDEN IN THE CENTRAL REGION OF RUSSIA: CHLAMYDIOSIS VS TRICHOMONIASIS. WHICH ONE IS THE LEADER?}

doi:10.1136/sextrans-2011-050108.4

T Ivanova, N Anisimova, A Guschin, E Simonova. Central Research Institute for Epidemiology, Moscow, Russian Federation

Background Infections caused by Chlamydia trachomatis (CT) are proved to lead to such dramatic complications as PID and infertility. That means that CT prevalence control is essential for arranging prophylactic actions. Official statistics claim trichomoniasis to be the most prevalent STI in Russia. For example, the amount of registered TV infections in 2009 was 1.8 times higher than the CT counts. Taking into consideration the absence of CT screening programs among general population along with the use of low sensitivity diagnostic tests (microscopy, DIF), it is reasonable to suppose that the real prevalence proportions are distinct from the official data. In this connection the aim of this study was to evaluate the STI prevalence pattern in a population sampling.

Methods This study included patients attending STD departments of outpatient clinics of Moscow region from 2004 to 2010. Total amount-190975 patients. For the evaluation of the STIs prevalence correlation samples from these patients we tested simultaneously for CT, GC and TV by PCR.

Results In 2004 a total of 23 (0.86\%) (95\% CI 13 to 32) TV positive results were obtained; 158 (5.89\%) (95\% CI 133 to 182) CT positive; infection proportion was 1:6.8 ( $p=0.05)$. In 2010 a total of 577 (1.1\%) (95\% CI 522 to 622) TV positive results were obtained; 2886 $(5.52 \%)(95 \%$ CI 2771 to 2980$)$ CT positive; infection proportion was 1:5 $(p=0.05)$. Annually this proportion did not vary dramatically and was no lower than 1:5 ( $\mathrm{p}<0.05)$. No significant diversity in the rates of TV and GC detection was observed except for 2009, when $521(1.22 \%$ ) (95\% CI 473 to 562$)$ tested positively for TV; 364 (0.85\%) (95\% CI 323 to 396 ) for GC, infection proportion was 1:1.4 $(\mathrm{p}=0.05)$ see Abstract P1-S1.04 Figure 1.

Conclusions This study shows that during the period 2004-2010 in a homogenous settings CT rates detected by sensitive methods were five or more times higher than TV rates. This might be an evidence of high CT prevalence and inappropriate official statistical recording of these infections in Russia.

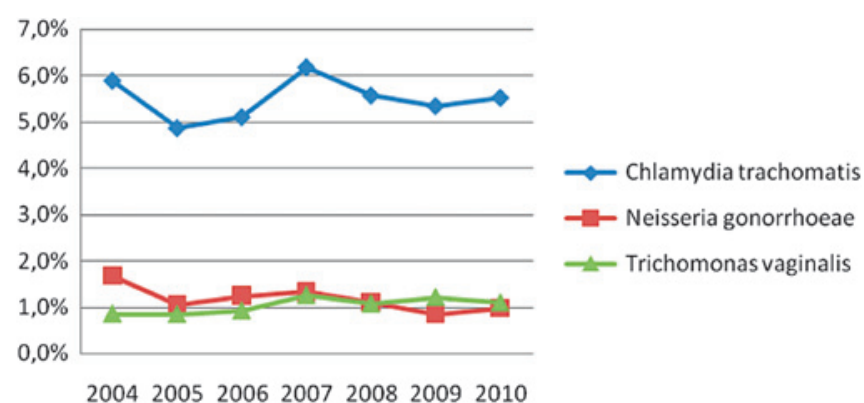

Abstract P1-S1.04 Figure 1 Prevalence of Chlamydia trachomatis, Neisseria gonorrhoeae, Trichomonas vaginalis 2004-2010.

\section{P1-S1.05 THE DETECTION RATE OF CHLAMYDIA TRACHOMATIS AND MYCOPLASMA GENITALIUM INFECTIONS IN STD CLINICS IN NOVOSIBIRSK, RUSSIAN FEDERATION}

doi:10.1136/sextrans-2011-050108.5

${ }^{1}$ A Khryanin, ${ }^{2} 0$ Reshetnikov. ${ }^{1}$ Novosibirsk State Medical University, Ministry of Public Health, Novosibirsk, Russian Federation; 2 Institute of Internal Medicine, Russian Federation

Background Currently, in Russia, the incidence of syphilis, gonorrhoea, chlamydia, trichomoniasis, urogenital herpes, and anogenital warts are officially registered. However, statistical records and reporting forms do not include mycoplasma infections (eg, Mycoplasma genitalium)

Methods The aim of the present study was to evaluate the detection rates of Chlamydia trachomatis and $M$ genitalium infections in patients who had attended to STD clinics in Novosibirsk in 2009-2010. A total of 9208 and 13006 patients were examined for $M$ genitalium and $C$ trachomatis, respectively, in different settings (antenatal clinics, hospitals, health centers, STI clinics). Both infections were tested in urethral and/or cervical swabs with nucleic acid amplification techniques ("Litex" and "DNA technology", Russia).

Results The detection rates of $M$ genitalium and $C$ trachomatis had not changed over 2009 and 2010, accounting to $12.6-12.6 \%$, and $12.9-13.0 \%$, respectively. Coinfection was observed in only $0.55 \%$ of examinies. However, seasonal variations showed different patterns for these two infections (Abstract P1-S1.05 figure 1). Statistical analysis by month revealed that the highest rates of $M$ genitalium were reported in February and March, and the lowest ones-in July. Monthly analysis found even distribution of infection with $C$ trachomatis along a year, while the lowest incidence was found in July.

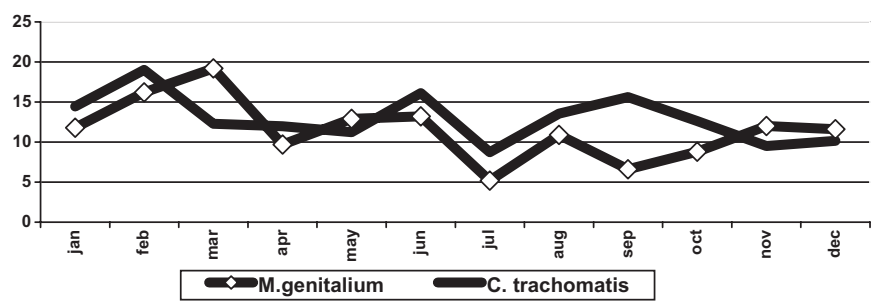

Abstract P1-S1.05 Figure 1 Detection rates of Mycoplasma genitalium and Chlamydia trachomatis according to the attendance data (by month in \%).

Conclusions The incidence rates of $C$ trachomatis and $M$ genitalium are approximately the same and account for $12-13 \%$ among men and women, equally. The combination of these infections is rare 